\title{
Assessment of the influence of astronomical cyclicity on sedimentation processes in the Eastern Paratethys based on paleomagnetic measurements using Discrete Mathematical Analysis
}

5 Boris Dzeboev ${ }^{1,2}$, Anastasia Odintsova ${ }^{1,3}$ and Alena Rybkina ${ }^{1,3}$

${ }^{1}$ Geophysical Center of the Russian Academy of Sciences (GC RAS), Moscow 119296, Russia

${ }^{2}$ Geophysical Institute - the Affiliate of Vladikavkaz Scientific Centre of the

3 Schmidt Institute of Physics of the Earth of the Russian Academy of Sciences (IPE RAS), Moscow 123995, Russia

Correspondence to: Anastasia Odintsova (a.odintsova@gcras.ru)

Abstract. The main objective of this study is to apply Discrete Mathematical Analysis (DMA) to the development of the methodology of cyclostratigraphy. This aim is supported by exploring the magnetic properties of rocks, the lithology of sediments and obtained geochronological reference definitions. The analysis was based on measurements of the variability of the magnetic susceptibility of rocks, which reflects climate variations. Astronomical cycles are global; this makes it possible to carry out a correlation analysis over a large area and on different facial types of sediments, considering their

20 lithology and other sedimentary features. The introduction of modern methods of mathematical processing of geological data is one of the prospective areas for investigation and development in geoscience. Astronomical cycles can be revealed from measurements of scalar magnetic parameters of rocks (magnetic susceptibility as presented by the authors). Specific software developed by the authors allows the processing of measurement data and assessment of the presence of stable oscillation cycles based on the obtained measurement base. The present study attempts to apply mathematical methods to magnetic data using the existing PAST program, which allows spectral analysis of primary data with the construction of Lomb-Scargle and REDFIT periodograms. We interpret the spectral analysis data based on paleomagnetic determinations, considering the available dates for the boundaries of direct and reverse polarity chrons on a general stratigraphic scale.

\section{Introduction}

In recent years, the rapid development of various techniques to study Earth processes has contributed to the generation of large amounts of heterogeneous data. Therefore, Earth Science urgently requires new methods and approaches to analyse these large datasets. This is especially true for describing complex phenomena and explaining some global events, such as paleoenvironmental changes, sedimentation rates, and fluctuations in insolation.

The Miocene stratigraphic scale of the Black Sea region is based on the determination of mollusc fauna, along with dates of the boundaries of regional stages and their subdivisions derived from various geochronology methods. However, some uncertainties remain. This problem provokes heated 
disputes and impedes the performance of full-scale interregional comparisons and paleogeographic reconstructions. Current efforts to solve the problem include improving the stratigraphic dissection of the studied sediments and modernising methods for dating rocks. These methods include one of the new directions in geology: cyclostratigraphy (dating of strata by astronomical cyclicity, recorded in scalar magnetic parameters), which is often used in combination with mathematical methods.

The authors propose integrating geological and mathematical methods to solve some critical

45 issues related to the cyclostratigraphy and paleogeography of Eastern Paratethys Miocene sediments. The present study describes the influence of astronomical cyclicity on the sedimentation processes of the Eastern Paratethys based on long-term fluctuations of insolation, cyclostratigraphy and paleomagnetic measurements, using Discrete Mathematical Analysis (DMA).

The cyclostratigraphy methods are based on assessing astronomical cyclicity and its recording in

50 sediments and accompanied by detailed lithological and paleomagnetic studies. Global fluctuations in insolation combined with traditional lithological methods allow an estimation of sedimentation rate and allow us to date the sediments. It is particularly important considering the lack of other methods to determine the absolute age of the sediments. The influence of astronomical cycles on sedimentation is global, meaning this method could be used for large-scale correlations.

55 Determination of astronomical cyclicity using geochronological methods, including paleomagnetic reconstruction and lithological analysis, is made possible by defining the magnetic susceptibility of sediments. Magnetic susceptibility depends on the amount of solar radiation reaching the Earth's surface and reflects climatic fluctuations. Further spectral analysis of scalar magnetic parameters allows the detection of long-term period oscillations of the Earth's axis, the angle of

60 inclination of the Earth's axis to the plane of the ecliptic, and eccentricity. This method can produce absolute ages of sediments with an accuracy on the order of 20,000 to 400,000 years.

The methodology proposed by the authors consists of synthesising lithological, paleomagnetic and mathematical methods for solving some cyclostratigraphy issues. The method has been validated on several sections of the Miocene sediments of Paratethys. The distribution of these outcrops on the East and Central Paratethys provides wide geographical coverage for comprehensive regional comparison and allows us to extend the methodology to other sections and outcrops accumulated under different conditions and regimes. Petromagnetic and paleomagnetic studies, including field measurements of the magnetic susceptibility of sediments using a field kappameter, are also significant component.

\section{Materials and Methods}

70 The stages of development of mathematical geology show a clear trend towards solving geological problems using increasingly complex mathematical models. Multidimensional methods are replacing simple statistics. The concept of stochastic processes changed our understanding of geological history and led to the development of geostatistics. Nonlinear models are replacing linear models, and the introduction of fractal sizes has led to the notion of chaotic behaviour. The methods used in

75 cyclostratigraphic studies utilise achievements in all of these areas. It should be noted that cyclic sequences are predictable [Schwarzacher, 1993].

Over the past 100 years, mathematical methods have evolved along with the available hardware, from simple computations in the early twentieth century to laborious mainframe algorithms in the midtwentieth century and the microcomputer revolution in the late twentieth century. The use of rapid 
80 algorithms on modern computers has made even the most complex multidimensional methods available to all researchers.

The most frequently used mathematical methods in geology can be roughly divided into three groups:

- Time series analysis, including spectral analysis (Fourier, spectrogram, and wavelet analysis), autocorrelation, cross-correlation, smoothing, filtering, and extremum search.

- Multivariate data analysis, including multivariate distributions and cluster analysis.

- Statistical methods, such as statistical distributions, correlation, regression, and chi-square tests.

Cyclostratigraphy is a new scientific domain in stratigraphy and sedimentology that deals with identifying, describing, correlating, and interpreting cyclic variations in the stratigraphic sequence. In

90 particular, cyclostratigraphy involves applying this knowledge to geochronology by increasing the accuracy and resolution of chronostratigraphic units. It uses the astronomical cycles of the currently known periodicity and an interpretation of the sedimentation conditions. Orbital cycles are the most important cycles. These cycles translate into climatic, oceanographic, sedimentary and biological changes recorded in sedimentary deposits over geological time. Numerous case studies have shown that

95 detailed analysis of sedimentary rocks allows these cycles to be identified with a high degree of confidence. An unprecedented high temporal resolution is available once the relationship between the sedimentary record and orbital forcing is established. This relationship provides a basis for timing the processes occurring in the Earth system [Strasser et al., 2006].

The main task of cyclostratigraphy is to analyse he structure of sedimentary deposits and

100 identify stable signals reflecting the orbital influence. An essential aspect of time series analysis in cyclostratigraphy is transforming raw data into the time domain using calibration points. Once the time series is established, mathematical methods can be used to detect orbital periodicities. Of the groups of mathematical methods listed above, the group 'Time series analysis', especially spectral analysis, is invaluable for solving cyclostratigraphy problems. A brief description of the spectral methods most commonly used in cyclostratigraphy is provided below.

Any time series can be analysed in terms of its description in the frequency domain. The classical way to detect frequency components in time series is Fourier spectral analysis. The importance of each frequency component in the time series is established using paired sine and cosine waves. Cyclostratigraphic data are usually discrete. For this reason, a discrete Fourier transform is applied. In a 110 discrete Fourier transform, a time series is multiplied, point by point, by a cosine wave of a specific frequency. The results are summed and multiplied by a constant $(2 / \mathrm{N}$, where $\mathrm{N}$ is the number of points in the data series). This calculation gives the average amplitude of the cosine frequency component. The calculations continue assuming half the spectrum exists as a mirror image of the actual spectrum at 'negative frequencies'. Since negative frequencies have no physical meaning for time series 115 observations, the average amplitude must be doubled using a constant. The time series is then multiplied by a sine wave of the same frequency. The results are again summed and multiplied by a constant. For each frequency component investigated, the relative size of the average amplitude of the sine and cosine waves determines the average phase of the time series oscillations. Fourier transform can be thought of as reorganising time series data to a different location based on frequency. 
To summarise, the Fourier transform is a mathematical operation that takes any waveform and breaks it down into separate sinusoidal components with different frequencies and amplitudes. The components are then presented as peaks in the frequency spectrum.

Spectral analysis of data series can be performed using the periodogram method. The periodogram is the square of the modulus of the amplitude of the Fourier spectrum. The Lomb 125 periodogram is a frequency analysis technique for non-uniform data series; it is more suitable for cyclostratigraphic data than the Fourier transform. The Lomb periodogram is invariant concerning the time scale shift, and its statistical properties for non-uniform samples are equivalent to the Fourier transform properties for uniform samples. Frequency analysis by the Lomb method solves the problem of detecting oscillatory processes in data series. However, to study the evolution of the observed phenomena requires a time-frequency analysis. In this analysis, a subset of the sample is selected by a sliding observation window. When using an observation window, the data series is multiplied by a specific weight function.

Time series spectra are often characterised by a continuous decrease in spectral amplitude with increasing frequency (red noise). Additionally, time series are often unevenly distributed in time, making it difficult to estimate their red noise spectra. The REDFIT mathematical method [Schulz and Mudelsee, 2002], an advanced version of the simple periodogram, solves this problem by directly fitting the first-order autoregressive process to unevenly distributed time series. In this way, the method avoids interpolation in the time domain and its inevitable shift. REDFIT is used to test if peaks in the spectrum of a time series are significant against a background of red noise from a first-order autoregressive process.

As mentioned above, knowledge of the periods can provide important information about the function and its phenomenon. Traditionally, spectral analysis was used to determine the period of a given function. At the Geophysical Center of the Russian Academy of Sciences (GC RAS), a novel technique has been developed, allowing assessment of the consistency of any positive number as a period of the original function. The most accurate answers, if they exist, will be the required periods.

The proposed technique is based on DMA [Agayan et al., 2018]. The new method is an original technique for analysing discrete data, developed at the GC RAS. DMA is a series of algorithms united by a common formal basis: fuzzy comparisons of numbers, a measure of proximity in discrete spaces, and a discrete limit. DMA was developed to create discrete equivalents of the concepts of classical mathematical analysis: for example, limit, continuity, smoothness, connectivity, monotonicity, and extremum. DMA methods and algorithms have proven to be useful in numerous studies related to the processing and analysis of various geological [Gvishiani et al., 2010], geophysical [Gvishiani et al., 2008a], geomagnetic [Bogoutdinov et al., 2010], seismological [Gvishiani et al., 2016] and other data [Gvishiani et al., 2008b].

155 In the following section, we provide a strict formulation of the problem and a description of the methodology. Suppose that a function $\mathrm{f}$ is given on the segment $[\mathrm{a}, \mathrm{b}]$, and we want to understand what periods it has. The period $\mathrm{T}$, ideal for $\mathrm{f}$, is defined as follows: $\mathrm{T}$ is the period for $\mathrm{f}$ if $\forall \mathrm{t}, \mathrm{t}+\mathrm{T} \in[\mathrm{a}, \mathrm{b}]$ is true for $f(t)=f(t+T)$. In reality, the function $f$ may not have any ideal period $T$, but it may have approximate periods. A description of approximate periods and how to determine them is provided below. 
It is clear that the applicant for the period $\mathrm{T}$ must lie in the interval $(0,(\mathrm{~b}-\mathrm{a}) / 2)$. For such a $\mathrm{T}$ and $\mathrm{t} \in[\mathrm{a}, \mathrm{a}+\mathrm{T})$, we denote by $\{\mathrm{f}, \mathrm{T}, \mathrm{t}\}$ the finite sequence $\{\mathrm{f}(\mathrm{t}), \mathrm{f}(\mathrm{t}+\mathrm{T}), \mathrm{f}(\mathrm{t}+2 \mathrm{~T}), \ldots\}$. Firstly, it is necessary to understand to what extent the sequence $\{\mathrm{f}, \mathrm{T}, \mathrm{t}\}$ can be considered constant, i.e., to determine the exponent of its constancy $\mathrm{C}\{\mathrm{f}, \mathrm{T}, \mathrm{t}\} \geq 0$. The equality $\mathrm{C}\{\mathrm{f}, \mathrm{T}, \mathrm{t}\}=0$ must correspond to the constancy of the sequence $\{f, T, t\}$.

Here we present three different constructions of the exponent $C\{f, T, t\}$. Firstly, the variance D of the sequence $\{f, T, t\}$ relative to the usual or average median:

$$
C\{f, T, t\}=D\{f, T, t\} \text { or } C\{f, T, t\}=D m\{f, T, t\} .
$$

The second and third constructions presuppose the determination of the modulus of the difference sequence $|\Delta\{f, T, t\}|$ for consistency $\{f, T, t\}$ :

$$
|\Delta\{f, T, t\}|=\{|f(t+T)-f(t)|,|f(t+2 T)-f(t+T)|, \ldots\} .
$$
$|\Delta\{\mathrm{f}, \mathrm{T}, \mathrm{t}\}|$.

The question of the constancy of $\{f, T, t\}$ is reduced to the question of the triviality of

The second construction is the Kolmogorov mean Mp with a positive exponent $\mathrm{p}$ of the sequence $|\Delta\{f, T, t\}|$ :

$$
C\{f, T, t\}=M p|\Delta\{f, T, t\}| .
$$

As mentioned previously, using the Kolmogorovsky mean $\mathrm{Mp}, \mathrm{C}$ indicates the proximity of the sequence $|\Delta\{\mathrm{f}, \mathrm{T}, \mathrm{t}\}|$ to zero.

Another solution is given by the third construction, using the distribution function $\mathrm{F}|\Delta\{\mathrm{f}, \mathrm{T}, \mathrm{t}\}|$ :

$$
C\{f, T, t\}=\alpha(|\Delta\{f, T, t\}|),
$$

where $\alpha(|\Delta\{f, T, t\}|)$ is $\alpha$-quantile of the distribution $F|\Delta\{f, T, t\}|$.

The estimate $C\{f, T, t\}$ characterizes $T$ as a period on the sequence $\{t, t+T, t+2 T, \ldots\}$.

The next stage is the development of a unified estimate $\mathrm{T}$ as a period independent of $\mathrm{t}$. Such an estimate $C(f, T)$ should be an indicator of the smallness of the collection $(C(f, T, t), t \in[a, a+T)$ ). For this, we again use the Kolmogorov mean Mp:

$$
C(f, T)=\operatorname{Mp}(C(f, T, t), t \in[a, a+T)), p \geq 0 .
$$

The final stage is the search for strong minima of the estimate C(f, T). Provided that they exist, we can obtain the necessary periods for the function $\mathrm{f}$ using the apparatus of minimality measures [Agayan et al., 2018].

\section{3. Results}

In the following section, we present the results of applying the above spectral methods to an example time series taken from [Lisiecki and Raymo, 2005]. The time series represents a series of oxygen isotopes of calcite in the shells of Pliocene and Pleistocene benthic foraminifera collected from 57 core samples worldwide. This series contains the last 400,000 years of the record in 1,000-year 190 increments (Figure 1). This series was chosen for demonstrating the application of spectral methods as it has been comprehensively studied. 


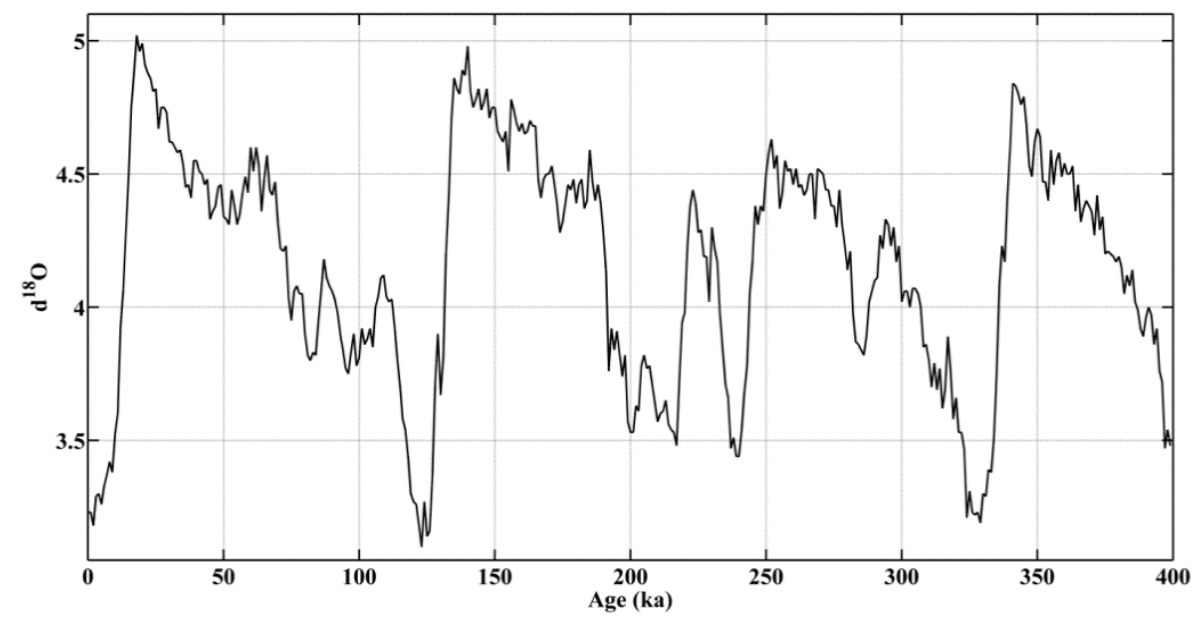

Figure 1: Temporal fluctuations of the isotopic composition of precipitation [Lisiecki and Raymo, 2005].

Figure 2a shows the Fourier and Lomb periodograms for the time series in Figure 1 [Lisiecki and Raymo, 2005], which was previously normalised and centred. The Fourier periodogram is shown in blue, the Lomb periodogram is shown in red [Hammer and Harper, 2005], and the spectrum obtained by the REDFIT method is shown in green [Hammer and Harper, 2005]. Let us consider the observed Milankovitch cycles. Figure 2 a shows that all six main peaks in the Fourier and Lomb periodograms are located at the frequencies (in decreasing order of spectral power): 0.0094, 0.0250, 0.0153, 0.0432, 0.0184, and 0.0339. This corresponds to the periods: 106,380 (eccentricity), 40,000 (tilt cycle), 65,360, 23,150 (precession), 54,350 and 29,500 years.

The coincidence of the frequency maxima of the Fourier and Lomb periodograms (Figure 2a) can be explained by several factors. Firstly, the initial data have a uniform temporal distribution of observations. For data such as these, in the absence of white noise, there is an identical coincidence of the Fourier and Lomba periodograms [Carbonell et al., 1992]. Secondly, the differences in periodograms are caused by the presence of minor noise in the original data. The Lomb method [Lomb, 1976] better distinguishes periods of 106,380 and 65,360 years from this noise.

The REDFIT spectrum (Figure 2b) shows five peaks at the frequencies (in descending order of 210 spectral power): $0.0094,0.0250,0.0163,0.0425$, and 0.0325 . Note that the two main peaks in the REDFIT spectrum are located at the same frequencies as the main peaks in the Fourier and Lomb periodograms. In this case, two REDFIT peaks are shifted in the frequency domain, and one peak combines two Fourier and Lomb peaks. The discrepancies may be because REDFIT considers the spectrum of red noise in the time series, which is not accounted for in the Fourier and Lomb transforms.

The results shown in Figure 2 demonstrate that spectral methods for studying time series are valuable tools in mathematical geology, particularly in cyclostratigraphy for detecting orbital periodicities. 


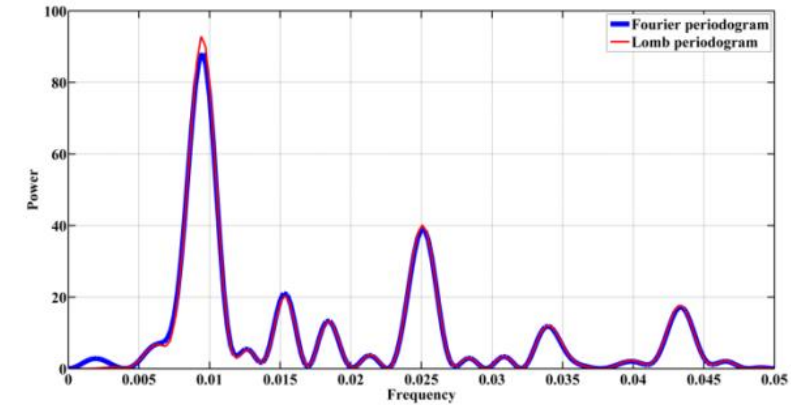

(a)

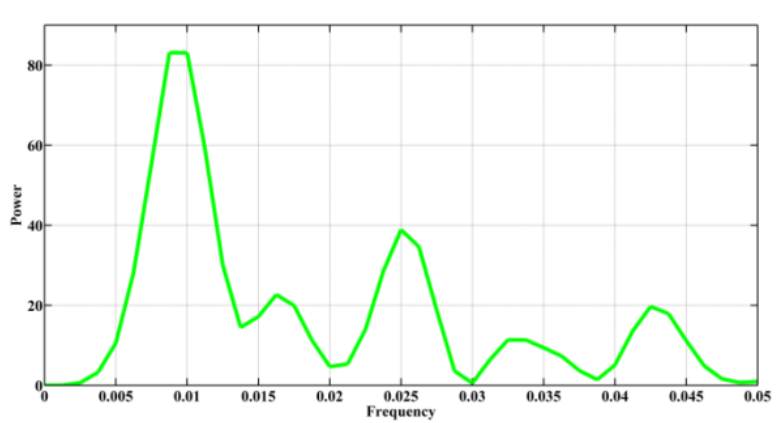

(b)

Figure 2: (a) Fourier and Lomb periodograms, and (b) REDFIT spectrum, constructed for the time series in Figure 1.

The efficiency of the new technique developed at the Geophysical Center of the Russian Academy of Sciences is demonstrated using the example of the time series shown in Figure 1. Figure 3 shows the calculated constancy exponent of the series in blue and its smoothed version in red. As mentioned previously, the required periods of the time series are the points of the minimum constancy exponent $\mathrm{C}(\mathrm{f}, \mathrm{T})$. Figure 3 shows that the strong periods are 115,000, 44,000 and 85,800 years. Weakly expressed periods are 58,600 and 67,600 years. Note that the identified periods are relatively close to the periods identified by spectral methods. Four out of five periods in Figure 3 closely match the periods in Figure 2. A possible explanation for the appearance of the 85,800-year period may be that it is a multiple of the period of 44,000 years.

The results shown in Figure 3 indicate that the proposed method for determining the periods of time series can be used simultaneously with spectral methods in practice.

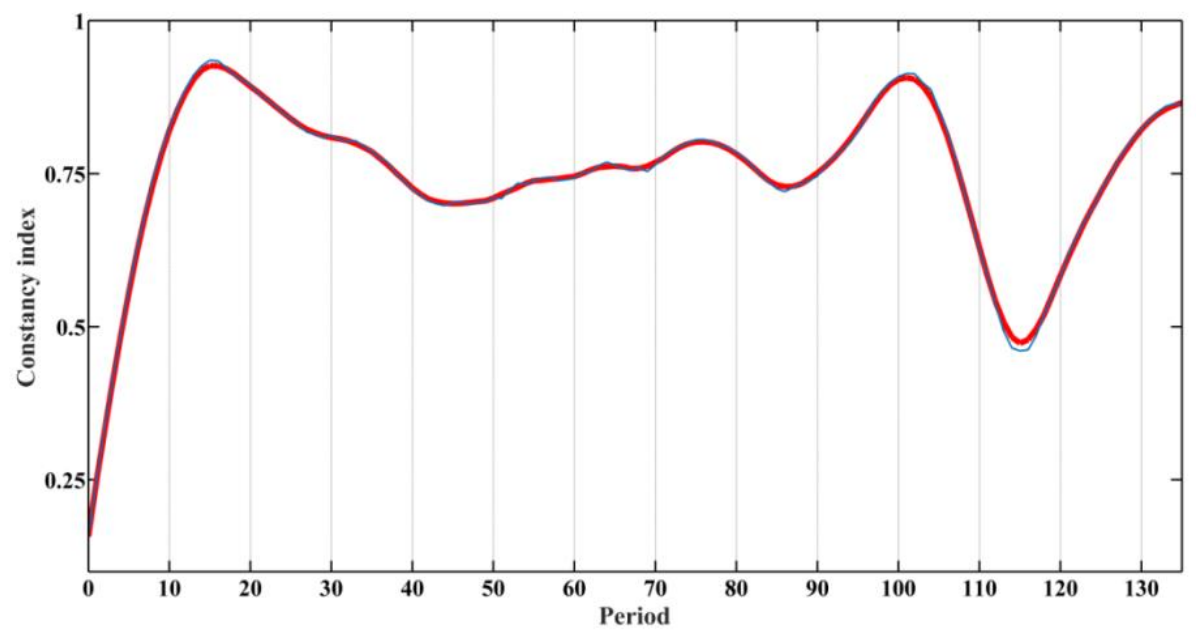

Figure 3: Function constancy exponent (construction - variance) and determination of periods 
We apply the above spectral methods, and the new approach developed at GC RAS to search for periods in magnetic susceptibility data of the Pontian (the total thickness of the sediments is $41.2 \mathrm{~m}$; 235 Figure 4a) and Lower Maeotian deposits (the total thickness of the deposits is $30 \mathrm{~m}$; Figure 4b) of Zhelezny Rog Cape. The section is located on the Black Sea coast of the Taman Peninsula (Russia) and is a reference section for the Pontian regional stage of the European part of Russia. Measurements of the magnetic susceptibility of rocks were carried out using a field kappameter across the strike of the layers at regular intervals of $20 \mathrm{~cm}$. Three measurements were taken at each point to ensure accuracy.

240 For each point, the average susceptibility was calculated from the three measured values. The averaged magnetic susceptibility values are shown in Figure 4.

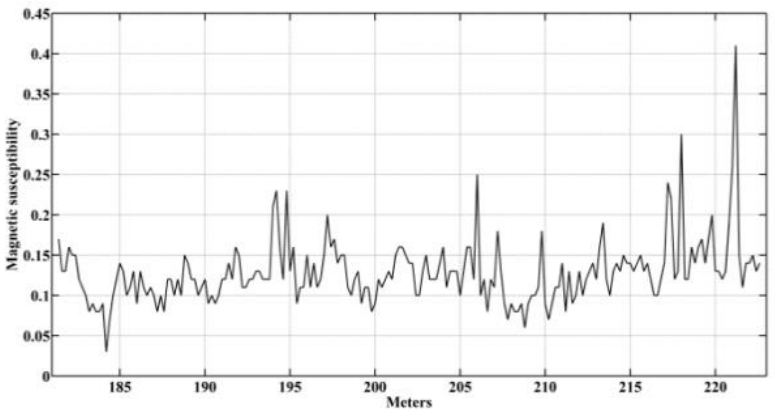

(a)

Figure 4: Magnetic susceptibility of deposits of the Zhelezny Rog Cape section: (a) Lower-Upper Pontian; (b) Lower Maeotian.

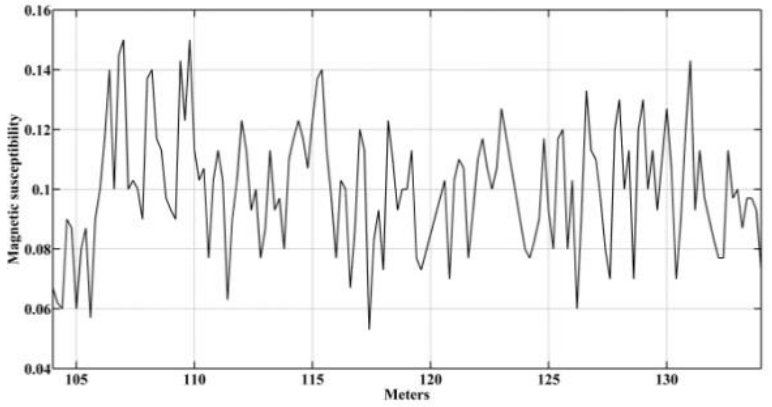

(b)

Figure 5 shows the results of applying spectral methods to the susceptibility data for the Pontian 245 deposits. Of the three prominent peaks on the Lomb periodogram, two peaks fall within the $95 \%$ confidence interval (Figure 5a). These peaks are located at frequencies of 0.0425 and 0.2519 , corresponding to periods of $23.53 \mathrm{~m}$ and $3.97 \mathrm{~m}$, respectively. This result is confirmed by the Fourier periodogram (Figure 5a), where the main peaks are located at frequencies of 0.0437 and 0.2519 periods of $22.88 \mathrm{~m}$ and $3.97 \mathrm{~m}$. In contrast to the example described above (Figure 2a), the Fourier and 250 Lomb periodograms in Figure 5a differ slightly. These differences can be explained by white noise in the original data (Figure 4a).

Figure $5 \mathrm{~b}$ shows the spectrum obtained using the REDFIT algorithm. Two peaks fall within the $95 \%$ confidence interval. They occur at frequencies of 0.0444 and 0.2525 , which correspond to periods of $22.52 \mathrm{~m}$ and $3.96 \mathrm{~m}$. These periods coincide with the periods identified by the Fourier and Lomb periodograms. 


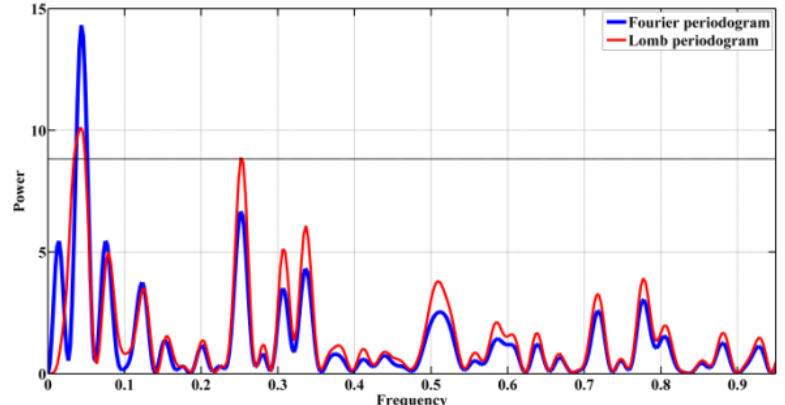

(a)

Figure 5: The results of applying spectral methods to the magnetic susceptibility data from the Lower-Upper Pontian deposits from the Zhelezny Rog Cape section (Figure 3a): a) Fourier and Lomb periodograms (the bold solid line indicates the 95\% confidence interval for the Lomb periodogram); b) the spectrum constructed by the REDFIT algorithm (the bold solid line indicates the $95 \%$ confidence interval).

Figure 6 shows the Fourier and Lomb periodograms and the REDFIT spectrum for the magnetic susceptibility data from the Lower Maeotian deposits. Only one peak of the Lomb periodogram falls within the $95 \%$ confidence interval (Figure 6a). This peak is located at a frequency of 0.1408 , which corresponds to a period of $7.1 \mathrm{~m}$. This result is partially confirmed by the Fourier periodogram, where the main peak is at a frequency of 0.1516 (period $6.6 \mathrm{~m}$ ). We are inclined to believe that the differences in the periodograms can be explained by noise in the data. The REDFIT spectrum is shown in Figure 6b. In this spectrum, just one peak is located in the $95 \%$ confidence interval. It occurs at a frequency of 0.1497 , corresponding to a period of $6.68 \mathrm{~m}$. The similarity in the periods obtained from the Fourier periodogram and the REDFIT spectrum may indicate more white noise than red noise in the initial data.

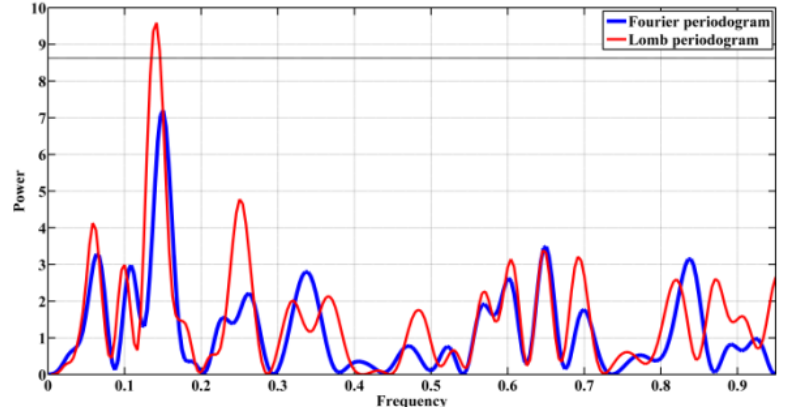

(a)

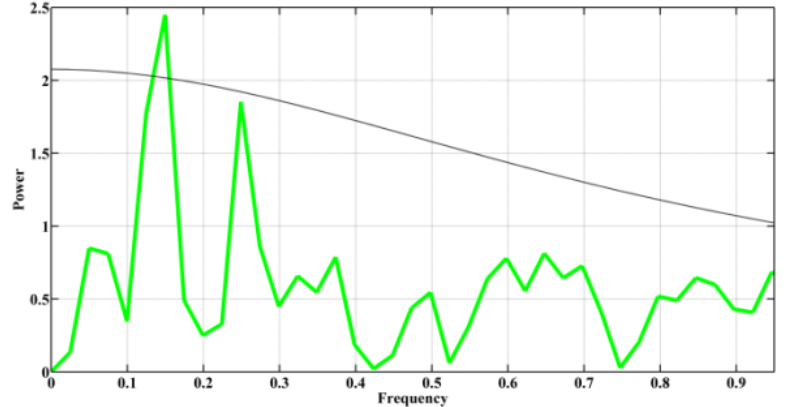

(b)

Figure 6: The results of applying spectral methods to the magnetic susceptibility data of the Lower Maeotian deposits of the Zhelezny Rog Cape section (Figure 3b): (a) Fourier and Lomb periodograms (the bold solid line indicates the 95\% confidence interval for the Lomb periodogram); (b) the spectrum constructed by the REDFIT algorithm (the bold dotted line indicates the $95 \%$ confidence interval).

We apply the new algorithm created in the Geophysical Center to selected periods. In Figure 7, the calculated constancy values for the magnetic susceptibility data of the Pontian and Lower Meotian 
deposits are shown in blue. As discussed previously, periods are considered to be the points of 'global' minima of the constancy exponent. For this reason, the calculated constancy exponents were 'strongly' smoothed to obtain their global trends. The smoothed constancy exponents are shown as red lines in Figure 7. Figure 7a shows that the prominent period in the magnetic susceptibility data series of the 280 Pontian deposits is $3.6 \mathrm{~m}$. The minimum of the constancy exponent at $15.2 \mathrm{~m}$ can be considered a 'weak' period. The absence of the $\sim 23 \mathrm{~m}$ period in Figure $7 \mathrm{a}$ is due to the algorithm considering values in half of the studied segment to be contenders for the period, which in this case is $41.2 \mathrm{~m}$. For the Lower Meotian deposits, only one period of $7 \mathrm{~m}$ can be distinguished (Figure 7b). Strong periods in Figure 7 emerge as very close to the periods obtained using spectral methods of analysing data series. In 285 the case of a 'softer' smoothing of the constancy exponent (Figure 7), weaker periods can be obtained. In Figures 5-6, these were not valid above the $95 \%$ confidence intervals or could not be obtained due to the low spatial resolution of the initial data.

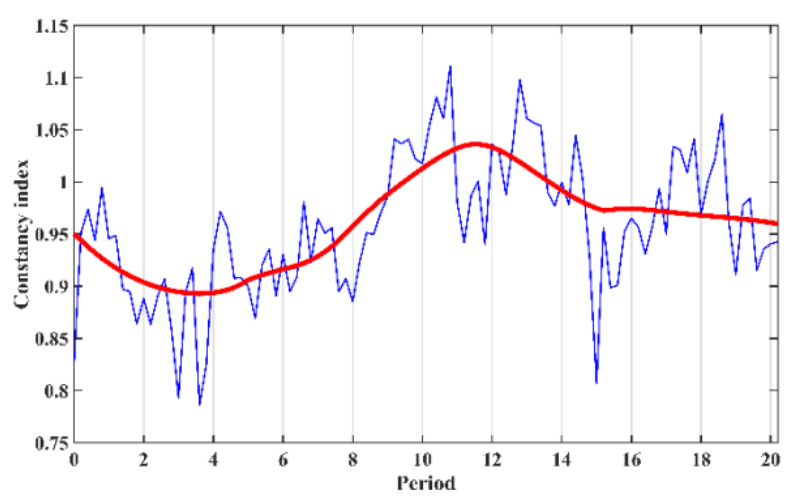

(a)

Figure 7: Determination of the periods in the magnetic susceptibility data: (a) Lower-Upper Pontian deposits; (b) Lower Maeotian deposits. The calculated constancy exponent of the series is shown in blue (construction - dispersion, see above). The 'strongly' smoothed version is shown in red.

\section{Conclusions}

This study presents new experimental data of the Pontian and Lower Maeotian of the Black Sea Basin (Paratethys) obtained by Time series analysis of magnetic susceptibility data from relatively deep-water sediments exposed in the Zheleznyi Rog Cape section (Taman Peninsula, Russia).

The DMA-based algorithm developed by the authors allows us to carry out the main tasks of cyclostratigraphy effectively. Periodograms were constructed to further identify repetitive cycles for carrying out geological reconstructions and correlating sediments of the same age over a large area. In the studied interval, a 71-m-long sedimentary sequence, spectral analysis revealed statistically significant signals with some highland peaks. These signals correspond to the precession and obliquity cycles. The $3.6 \mathrm{~m}$ peak corresponds to the precession periodicity (19-24 thousand years). The $7 \mathrm{~m}$ peak corresponds to the periods of changes in the angle of inclination of the Earth's axis (41,000 years). The 15.2 m peak corresponds to 100,000-year cycles; however, its validity is questionable due to the length of the interval $(15 \times 3=45 \mathrm{~m})$. The $23 \mathrm{~m}$ peak is not valid, as the sampling interval is $41.2 \mathrm{~m}$ (cycle 
lengths are valid when they are three times the thickness of the interval: $23 \times 3=69 \mathrm{~m}$ ). The analyzed parameters of the magnetic susceptibility of the Zhelezny Rog section are consistent with the data from the study of these strata using the Past and AnalySeries programs, the selection of astronomical cycles was carried out [Rybkina and Rostovtseva, 2014; Rostovtseva and Rybkina, 2017].

This study correlates the main steps of Messinian Salinity Crisis (MSC) of the Mediterranean to the Black Sea Pontian record based on astronomical tuning of the study sequence and evaluation of integrated biostratigraphic, paleomagnetic and sedimentological data [Hsü et al., 1973].

It is necessary to say in conclusion a few words, about the mathematical apparatus of DMA [Agayan et al., 2018]. It is being developed at the Geophysical Center of the Russian Academy of Sciences and forms the basis of the methodology for searching periods in the series of cyclostratigraphic data presented in the article. It is important to emphasize that DMA is a direction of modern applied systems analysis [Zgurovsky and Pankratova, 2007].

DMA has all the necessary tools to generate mining algorithms for geological and geophysical data, including searching for hidden periods / cycles. Based on fuzzy sets and fuzzy logic, DMA has the ability to convey expert ideas about the structure, morphology, monotony, and other of studied data series. Thus, DMA enables a systematic approach to the analysis of complex data series of Earth 320 sciences.

The Geophysical Center of the Russian Academy of Sciences has a vast experience of application DMA-structures for solving various geophysical problems. Among them are recognition of low-amplitude geomagnetic pulsations of various frequency ranges from 1 to $30 \mathrm{MHz}(\mathrm{Pc} 3, \mathrm{Pi}, \mathrm{Pc} 5)$ on records of geomagnetic field variations; recognition of anomalies on magnetic records of the

325 INTERMAGNET network; recognition of areas prone to strong earthquakes; recognition of anomalies in the records of residual gravity field variations of the global network of superconducting gravimeters GGP Network; recognition of anomalies on the records of the intrinsic electric potential of the Piton de la Fournaise volcano (Reunion Island, France); identifying areas of stability in radar interferometry images for monitoring the activity of Mount Etna (Sicily); analysis of a complex interference magnetic

330 field in the Gulf of Saint-Malo (Brittany, France); direction assessment of the magnetization vector for geological bodies in the area of the Ahaggar massif (Algeria) and others [Bogoutdinov et al., 2010; Gvishiani et al., 2008a; Gvishiani et al., 2002; Zelinskiy et al., 2014; Soloviev et al., 2005; Zelinskiy et al., 2014; Soloviev et al., 2015; Gvishiani et al., 2003; Gvishiani et al., 2004; Kulchinsky et al., 2010; Mikhailov et al., 2003; Zlotnicki et al., 2005]. Thus, the results of this work show that DMA is also applicable for solving cyclostratigraphy problems.

\section{Code availability}

The code presented in this study is available on request from the corresponding author. The code is temporarily not publicly available due to research policies and implementing research programmes.

\section{Data availability}

The data presented in this study are available on request from the corresponding author. The data are temporarily not publicly available due to research policies and implementing research programmes. 


\section{Author Contributions}

345 Conceptualization, A. R., B. D. and A. O.; Data curation - A. O. and A. R.; Methodology, A. R., B. D. and A. O.; Software, B. D.; Formal analysis - B. D.; Validation - A. R.; Resources, A. R.; Writing Original Draft Preparation, B. D. and A. O.; Writing - Review \& Editing, B. D., A. O. and A. R.; Supervision, A. R.; Project Administration, A. R. and A. O.; Funding Acquisition, A. R.

\section{Conflicts of Interest}

350 The authors declare no conflict of interest.

\section{Acknowledgments}

The authors are grateful to employees of the Geophysical Center of the Russian Academy of Sciences Sergey Agayan, Shamil Bogoutdinov, Dmitry Kudin, and Boris Dzeranov for assistance with developing the algorithm and implementing the program code.

\section{References}

Agayan, S. M., Bogoutdinov, Sh. R., Krasnoperov, R. I.: Short introduction into DMA, Russian Journal of Earth Sciences, 18, ES2001, DOI: 10.2205/2018ES000618, 2018.

Bogoutdinov, Sh. R., Gvishiani, A. D., Agayan, S. M., Soloviev, An. A., Kihn, E.: Recognition of disturbances with specified mor-phology in time series. Part 1: Spikes on magnetograms of the

360 worldwide INTERMAGNET network, Izvestiya, Physics of the Solid Earth, 46, 1004-1016, DOI: 10.1134/S1069351310110091, 2010.

Carbonell, M., Oliver, R., Ballester, J. L.: Power spectra of gapped time series: a comparison of several methods, Astronomy and Astrophysics, 264, 350-360, 1992.

Gvishiani, A. D., Mikhailov, V. O., Agayan, S. M., Bogoutdinov, Sh. R., Graeva, E. M., Diament, M.,

365 Galdeano, A.: Artificial intelligence algorithms for magnetic anomaly clustering, Izvestiya, Physics of the Solid Earth, 38, 545-559, 2002.

Gvishiani, A. D., Agayan, S. M., Bogoutdinov, Sh. R., Bonnin, J.: New mathematical approach to cluster and classification analysis of potential field anomaly data, Conseil de l'Europe: Cahiers du Centre Europeen de Geodynamique et de Seismologie, 20, 29-34, 2003.

370 Gvishiani, A. D., Agayan, S. M., Bogoutdinov, Sh. R., Tikhotsky, S. A., Hinderer, J., Bonnin, J., Diament, M.: Algorithm FLARS and recognition of time series anomalies, System Research \& Information Technologies, 3, 7-16, 2004.

Gvishiani, A., Agayan, S., Mikhailov, V., Bogoutdinov, Sh.: Automatic fuzzy-logic recognition of anomalous activity on long geophysical records: Application to electric signals associated with the

375 volcanic activity of la Fournaise volcano (Reunion Island), Earth and Planetary Science Letters, 234, 261-278, 2005.

Gvishiani, A. D., Agayan, S. M., Bogoutdinov, Sh. R.: Discrete mathematical analysis and monitoring of volcanoes, Inzh. Ekol., 5, 26-31, 2008a.

Gvishiani, A. D., Agayan, S. M., Bogoutdinov, Sh. R., Zlotnicki, J., Bonnin, J.: Mathematical methods

380 of geoinformatics. III. Fuzzy comparisons and recognition of anomalies in time series, Cybernetics and Systems Analysis, 44, 309-323, DOI: 10.1007/s10559-008-9009-96, 2008b 
Gvishiani, A. D., Agayan, S. M., Bogoutdinov, Sh. R., Soloviev, A. A.: Discrete mathematical analysis and applications in geol-ogy and geophysics, Vestn. KRAUNTs, Nauki Zemle, 2, 109-125, 2010.

Gvishiani, A. D., Dzeboev, B. A., Agayan, S. M.: FCAZm intelligent recognition system for locating areas prone to strong earth-quakes in the Andean and Caucasian mountain belts, Izvestiya, Physics of the Solid Earth, 52, 461-491, DOI: 10.1134/S1069351316040017, 2016.

Hammer, Ø., Harper, D.A.T.: Paleontological Data Analysis, Blackwell Publishing, 351, DOI: 10.1002/jqs. 1107, 2005.

Hsü, K.J., Cita, M.B., Ryan, W.B.F.: The origin of the Mediterranean evaporates, Deep Sea Initial 390 Reports, 13, 1203-1231, 1973.

Kulchinsky, R. G., Kharin, E. P., Shestopalov, I. P., Gvishiani, A. D., Agayan, S. M., Bogoutdinov, Sh. R.: Fuzzy logic methods for geomagnetic events detections and analysis, Russian Journal of Earth Sciences, 11, DOI:10.2205/2009ES000371, 2010.

Lisiecki, L. E., Raymo, M. E.: A Pliocene-Pleistocene stack of 57 globally distributed benthic $\delta 180$ 395 records, Paleoceanogra-phy, 20, 1-17, DOI: 10.1029/2004PA001071, 2005.

Lomb, N.R.: Least-squares frequency analysis of unequally spaced data, Astrophysics and Space Science, 39, 447-462, 1976.

Mikhailov, V., Galdeano, A., Diament, M., Gvishiani, A., Agayan, S., Bogoutdinov, Sh., Graeva, E., Sailhac, P.: Application of artificial intelligence for Euler solutions clustering, Geophysics, 68, 168$400180,2003$.

Schwarzacher, W.: Mathematical geology and the development of cyclostratigraphy, Geoinformatics, 4, 353-356, 1993.

Strasser, A., Hilgen, F. J., Heckel, P. H.: Cyclostratigraphy - Concepts, definitions and applications, Newsletters in Stratigra-phy, 42, 75-114, DOI: 10.1127/0078-0421/2006/0042-0075, 2006.

405 Rybkina, A. I., Rostovtseva, Yu V.: Astronomically-tuned cyclicity in Upper Maeotian deposits of the Eastern Paratethys (Zheleznyi Rog Section, Taman), Moscow University Geology Bulletin, 69, 341346, 2014.

Rostovtseva, Yu. V., Rybkina, A. I.: The Messinian event in the Paratethys: Astronomical tuning of the Black Sea Pontian, Marine and Petroleum Geology, 80, 321-332, DOI: 410 10.1016/j.marpetgeo.2016.12.005, 2016.

Soloviev, An. A., Shur, D. Yu., Gvishiani, A. D., Mikhailov, V. O., Tikhotskii, S. A.: Determination of the magnetic moment vector using cluster analysis of the local linear pseudoinversion of $\Delta \mathrm{T}$ anomalies, Doklady Earth Sciences, 404, 1068-1071, 2005.

Zelinskiy, N. R., Kleimenova, N. G., Kozyreva, O. V., Agayan, S. M., Bogoutdinov, Sh. R., Soloviev,

415 A. A.: Algorithm for recognizing Pc3 geomagnetic pulsations in 1-s data from INTERMAGNET equatorial observatories, Izvestia-Physics of the Solid Earth, 50, 240-248, 2014.

Zgurovsky, M. Z., Pankratova, N. D., System Analysis: Theory and Applications, Springer-Verlag Berlin Heidelberg: Data and Knowledge in a Changing World, 447, DOI: 10.1007/978-3-540-48880-4., 2007.

420 Zlotnicki, J., LeMouel, J.-L., Gvishiani, A., Agayan, S., Mikhailov, V., Bogoutdinov, Sh.: Automatic fuzzy-logic recognition of anomalous activity on long geophysical records: Application to electric signals associated with the volcanic activity of la Fournaise volcano (Reunion Island), Earth and Planetary Science Letters, 234, 261-278, 2005. 